\title{
Relativity without miracles
}

\section{Adán Sus}

\begin{abstract}
It has been claimed, recently, that the fact that all the non-gravitational fields are locally Poincaré invariant and that these invariances coincide, in a certain regime, with the symmetries of the spacetime metric is miraculous in general relativity (GR). In this paper I show that, in the context of GR, it is possible to account for these so-called miracles of relativity. The way to do so involves integrating the realisation that the gravitational field equations (the Einstein field equation in GR) impose constraints on the behaviour of matter in a novel interpretation of the equivalence principle, which dictates the determination of local inertial frames through gravitational interaction. This proposed explanation of the miracles can also deal with the problematic cases for attempts at explaining them in the context of the standard geometrical perspective on relativity theory.
\end{abstract}

\section{Introduction}

What is postulated and what is derived in a physical theory is probably not an absolute matter: claims about the fundamentality or derivative character of some features denote different stances on the interpretation of the theory. Recently, it has been claimed that the fact that all the matter laws have the same local symmetries (local Poincaré invariance) and that these coincide with the symmetries of the spacetime metric is miraculous in general relativity (GR). These two unexplained, and highly surprising, coincidences - of all the local symmetries of the matter laws and of them with the spacetime symmetries

Adán Sus

Dept. of Philosophy

University of Valladolid

Tel.: Fax:

E-mail: adansus@fyl.uva.es

Forthcoming in the European Journal for the Philosophy of Science PLEASE CITE THE PUBLISHED VERSION 
- are called in [40] the two miracles of relativity. My aim in this paper is to argue that relativity theory, at least under a certain interpretation, has the resources to account for these coincidences: to show that they can be seen as somehow derived instead of brute facts and therefore not as miraculous after all. As I will discuss, this does not mean that there is no contingent component in them.

Let me be more specific. [40, 38] present the problem in the following way. Brown's influential dynamical approach attempts to regard spacetime in special relativity (SR) as a codification of certain facts about the laws governing the matter fields. As a consequence of this, the symmetries of the metric are seen as accounted for by the Poincaré invariance of matter laws which is seen, consequently, as a brute fact in SR. In GR things are a bit more complicated. The fact that the metric is itself a dynamical field seems to make the previous interpretation untenable. Nevertheless, the dynamical perspective can endorse the view that the chronogeometrical significance of the metric, the fact that rods and clocks survey a metric that is a solution of Einstein's field equation, itself a consequence of the equivalence principle, is also an unexplained coincidence in GR. These are the grounds in which the two miracles naturally appear for the dynamical perspective on relativity.

My starting point is that an interpretation in which those mysteries appear as derived from, and somehow explained by, some principles of relativity theory is preferable. Such principles might or might not be common to other theories but, in any case, are part of the foundations of theories that have something to do with spacetime. The aim of this paper is then to present a strategy that allows one to insert the alleged miracles of GR into a scheme in which, at least, their miraculous status is, if not completely dissolved, greatly diminished.

The key novel idea to be developed in my discussion is that it is the equivalence principle, under a certain interpretation, which can do the work of explaining the miracles in GR. A first reaction to a proposal of this type is that a principle can only explain insofar as it is itself justified. In fact, Read, Brown and Lemhkuhl 40] discuss the role of the equivalence principle in the formulation of the miracles and rather than as a solution, they see it as a way of expressing the miraculous status of these features of relativity. Brown's dynamical perspective on relativity takes the strong equivalence principle (SEP) to be responsible for the chronogeometrical significance of the metric and the above-mentioned discussion in [40] makes explicit that the content of SEP is nothing else than what they claimed to be miraculous in relativity. One of the main tasks of my following discussion is to make clear how to understand the equivalence principle in order to regard it as the expression of an explanation instead of as the expression of an unexplained coincidence.

This is how. As I will discuss in 4.1, the SEP is usually taken as a condition on the local form of non-gravitational laws and the motion of free-bodies. If this is all there is to it, clearly the principle is just a statement of the miracles under a fancy name. Nevertheless, one can claim that what should be central to the principle, and makes its physical content more specific, is its connection to the gravitational interaction. Basically, the principle is intended to claim 
that the conditions on the default behaviour of matter are somehow imposed by or through the gravitational interaction. Taking this into account, we have a potential explanation of the miracles by regarding the conditions on the local invariance of laws and the motion of force-free bodies as determined through the dynamical equations that describe gravity. Irrespective of whether it is advisable to see this as part of the equivalence principle, my claim is that in GR (and also some other theories) there are gravitational field equations that impose constraints on the behaviour of matter (non-gravitational fields) and that this can be seen as an, at least partial, explanation of the miracles of relativity.

Let me advance the result that supports this claim of the explanation of the miracles in GR. I will show specifically (in [5.2]) in what sense the satisfaction of the Einstein field equation imposes constraints on the dynamics of non-gravitational fields. This is based, first, on the possibility of deriving the (covariant) conservation of the energy-momentum tensor from the equations for the gravitational field and, second, on extracting from it approximate geodesic motion and the local Poincaré invariance of the equations for the nongravitational fields. As I will argue, all this supports the claim that the validity of Einstein's field equation somehow constrains matter behaviour, even recognising that the conservation condition can be derived independently of the equation and that there are certain assumptions that enter into the derivation of local Poincaré invariance and that introduce important qualifications. I will claim that this is enough to explain the coincidence of the local symmetries of all matter laws and of these with the local symmetries of the metric in the context of GR, as expressed by the miracles.

The plan of the paper is as follows. First I introduce the miracles 2 and discuss their logic 3 in order to make explicit what role principles like minimal coupling and the equivalence principle play in the formulation of the miracles 4. The aim of this first part of the paper is to state as clearly as possible what assumptions and interpretational commitments enter in claiming that these facts about the local invariance of laws is miraculous and why no promising explanation is considered possible in GR. The second part of the paper is dedicated to presenting my proposed account of the miracles. In [5], I propose an interpretation of the equivalence principle that, I argue, contains an explanation of the miracles. This includes a discussion of in what sense the gravitational field equations in GR can be thought of as imposing constraints on matter behaviour [5.2] and how this fact is part of an explanation of the miracles 5.3. I also discuss in [6] how my proposal deals with theories that are problematic for an attempt to explain the miracles that would regard the coincidence of invariances of non-gravitational fields as a reflection of the fact that they all couple to the same metric because it represents the spacetime in which all these fields live. I conclude making explicit how this explains the alleged miracles of relativity. 


\section{The two miracles}

Before focusing on the arguments given for the existence of the miracles of relativity, we need to be clear about what the claims say. 40, 38 contain their rigorous exposition. Nonetheless, for the sake of self-containment, I will summarise here the miracle claims, in order to show the logic and assumptions of the arguments that support them.

This discussion can be seen partly as a development of the dispute between the so-called dynamical and geometrical approaches to the interpretation of SR (henceforth, DA and GA respectively). Brown's highly influential book Physical Relativity [6] presents a perspective on SR, extendible to other theories, in which spacetime structure is ontologically dependent on matter laws and spacetime symmetries are explained by the dynamical symmetries of such laws. This is opposed to the standard view, the geometrical approach (GA), which conceives spacetime as ontologically primary and the symmetries of laws as explained by the symmetries of spacetime structure 1 In this streamlined version of the dispute, it is already clear that the DA, in opposition to the GA, seems to assume that, in a theory like SR, all matter laws happen to have the same symmetries as a matter of fact, while allegedly being explained by the symmetries of the spacetime metric for the GA. This is the origin of the first miracle of relativity. The DA involves the claim that the coincidence of the dynamical symmetries of all matter laws, in SR, is a cosmic coincidence; the GA claims to provide an explanation of it, although its efficiency in doing so has been repeatedly questioned ${ }^{2}$ With this in mind, the first miracle of relativity can be formulated.

MR1: All non-gravitational interactions are locally governed by Poincaré invariant dynamical laws ${ }^{3}$

The local qualification of the first miracle is motivated by considerations coming from GR, where the metric field does not necessarily have global symmetries. Moreover, as everybody agrees, the ontological status of the metric is quite different in that theory. The DA can hold that Minkowski metric in $\mathrm{SR}$ is just a codification of certain features of the dynamical laws, while the default version of the geometrical approach sees the metric as an ontologically distinct entity. ${ }^{4}$ But this ontological reduction of the metric is more difficult to maintain in GR, where the spacetime metric is a full dynamical player and its reduction to matter fields seems loaded with problems. In view of this, the DA accepts, like the GA, the ontological autonomy of the metric, which means that it may be possible that its symmetries be different from the symmetries

1 Examples of the geometrical approach can be found in [31, 25.

2 Critical discussions of the limits of the GA can be found in [44, 39, 1], 32, 8

3 [40, p. 20.

4 Janssen's version of GA (see 25) claims to be ontologically neutral and not to imply commitment to a sustantivalist perspective. The merits of this version of GA and its success in maintaining the ontological neutrality while providing a distinctive perspective is discussed in 44. 
of the dynamical laws. The fact that locally the symmetries of matter laws coincide with the symmetries of the metric in GR provides, then, the second miracle of relativity. This is the formulation given in [40]

MR2: The Poincaré symmetries of the dynamical laws governing nongravitational fields in the neighbourhood of any point $p \in M$ coincide - in the regime in which terms featuring the Riemann tensor or its contractions may be ignored - with the symmetries of the dynamical metric field in that neighbourhood.

\section{The logic of the miracles}

Let us state as clearly as possible the logic behind the miracles claims. The situation concerning SR is pretty clear: for the DA, that all matter laws are Poincaré invariant is a brute fact $5^{5}$ One might think of this as a defining, unrenounceable, posit of the DA. If the DA takes matter laws as ontologically primitive and sees spacetime structure as a codification of certain features of such laws, it is natural to regard their symmetries as brute unexplainable features. This seems to assume the following dilemma: either symmetries of laws are explained by spacetime symmetries (the defining maxim of the GA) or they must be taken as an unexplained coincidence. Even if these are not the only options available in SR, the higher complexity of the formulation of the miracles in GR provides a vantage point to treat the situation in the two theories conjunctly ${ }^{6}$ So, before questioning the claim in SR, it seems advisable to review the situation in GR.

As I said above, things get more complicated in GR. First we have the question about ontology: given the nature of the theory, in which the spacetime metric is determined through field equations with the possibility of having vacuum solutions, the DA faces difficulties in implementing a programme of reducing spacetime to features of matter laws. This leads the DA to introduce the second miracle (MR2) in GR. Furthermore, the metric in GR does not posses global symmetries that hold in all the solutions. This forces one to localise the validity of the claims about the coincidence of symmetries to appropriate neighbourhoods. These two facts are reflected in the formulation of the DA in the context of GR. The core of this perspective consists in providing an account of how the metric acquires its chronogeometric significance in the theory. Brown's original maxim is that it "earns its spurs by way of the strong equivalence principle" [6. This somehow cryptic claim must be clarified, and it is so, by saying what is meant by "strong equivalence principle" and making explicit in what sense this affords the chronogeometrical character to the metric in a way that it is consistent with the spirit of the DA.

The basic idea is simple but imprecise: in GR the equations for the nongravitational fields reduce locally to its special relativistic form. So the mentioned strong equivalence principle involves a claim about the couplings of

${ }^{5}$ Claims in this sense can be found in [6, 9], 40, 39]

6 A similar claim can be found in [36]. 
matter (non-gravitational) fields to the metric: it must be such that their equations reduce to the ones that would govern the field in SR. But in this claim there are a number of ambiguities that must be resolved. First, the statement about coupling is usually expressed in a recipe called minimal coupling. It prescribes how one is to construct general relativistic matter field equations starting from special relativistic ones. Basically the procedure consists in replacing, in the equations, the fixed Minkowski metric $\left(\eta_{a b}\right)$ with the metric determined by Einstein field equations $\left(g_{a b}\right)$ and the derivative operator compatible with $\eta_{a b}$ with the one compatible with $g_{a b}$. This seems easy but, as 40 show for the case of minimally coupled Maxwell electromagnetism, it has the, perhaps unwanted, consequence of generating second order equations in which curvature terms appear. So if one understands minimal coupling as a way of generating equations that, at a point, recover their special relativistic form, this case seems to go against it. Another way to put the point: the recipe of minimal coupling is not as well defined as it might seem as there are equations in which it is always possible to include curvature terms; if one understood minimal coupling as forbidding such terms, in order to always be able to recover the special relativistic form at each point, the question would be whether to apply it in this way and to which equations; and also what justifies one in doing so when it is possible to generate equations in which curvature terms are present. The answer to these questions is connected to the interpretation of the equivalence principle and to considerations about the approximate validity of the claims.

With these elements, one can formulate a version of the DA that is valid in GR by giving specific content to the idea of the metric earning chronogeometrical significance through the strong equivalence principle (SEP). Of the different possible formulations of the strong equivalence principle, one that seems operative in GR is what is labeled as EP1' in [40]:

EP1': The dynamical equations for non-gravitational fields reduce to a Poincaré invariant form, with no terms featuring the Riemann tensor or its contractions, in a neighbourhood of any $p \in M$.

40. discuss different formulations of the SEP: EP1 claims the reduction of dynamical equations to Poincaré invariant form with no terms featuring the Riemann tensor at any point while the prime version (EP1') extends its validity to a certain neighbourhood of any point. There are weaker versions of those (EP2 and EP2') that omit the mention to the Riemann tensor and would be valid whenever EP1 and EP1' are so, respectively. It must be stressed that all these versions of SEP, except the extremely weak EP2, hold only approximately in GR. The problem with EP1 and EP1', as [40] mention, is that it is incompatible with some minimally coupled equations. Having said that, discussion of EP1' will be enough for our purposes.

The equivalence principle captures the idea of the local validity of SR. EP1' ensures that in a neighbourhood, whose determination will depend on the available experimental apparatuses not detecting the curvature terms, the nongravitational equations are Poincaré invariant. Moreover, locally, any metric 
solution of the Einstein field equations has Poincaré symmetries $]^{7}$ This means that, conditional on EP1' holding, all the non-gravitational laws, in those neighbourhoods in which curvature terms can be ignored according to our measuring devices, are equally Poincaré invariant and the devices that are governed by such laws seem to survey the metric field of the theory ${ }^{8}$ This is then the content of the claim quoted above: the metric field earns is spurs (its chronogeometrical meaning) by way of the strong equivalence principle (understood as EP1') ${ }^{9}$ But if this is the case, the claim seems equivalent to positing the two miracles (MR1 and MR2) of relativity.

Finally, now we are in the position of making fully explicit the logic of the miracles in GR. One way to put it is by saying that EP1' holds in GR and noticing that this is a fancy way to express the local special relativistic character of non-gravitational laws (with all the mentioned caveats relative to neighbourhoods and curvature terms). The question then is: What reasons do we have to expect that something like some version of the strong equivalence principle is true? The answer in tune with the miracles view is: none ${ }^{10}$ As I will defend below, this position contains the seeds to explain away (or dissolve) the miracles, but involves interpreting the strong equivalence principle differently.

A more explicit way to support the claim of the miracles in GR is by taking something close to minimal coupling as the primitive brute fact in GR which constrains the laws to be locally Poincaré invariant ${ }^{11}$ Even more ex-

7 It must be noted that the form of the Einstein field equation does not determine the signature of the metric. As Brown stresses when discussing the chronogeometrical significance of the metric, inspired by Anderson's formulation of the equivalence principle (see 6, section 9.2.3), according to the form of the equations the signature of the metric is indeterminate. At the end of the paper I will have more to say on the significance of this fact.

8 See the development of this argument in [40, section 4.1

9 Claims like this one, pertaining to the original formulation of the DA, might be too crude: the chronogeometrical significance of the metric must presuppose, beyond the SEP, the existence of stable rods and clocks. According to this, the structure recovered through the equivalence principle is what 41 call theoretical spacetime, distinct from the operational spacetime that would presuppose the existence of stable rod and clocks.

10 The question and its answer here refer to theoretical reasons; without any doubt, the miracles view admits empirical reasons for the validity of SEP.

11 Further clarification about the relation of minimal coupling and SEP seems necessary. As it is implicit in the presentation of the different versions of SEP, the authors of [40] do not think that any version of SEP is incompatible with non-minimal coupling; in fact, they show in that paper that it is possible to have non-minimally coupled equations in GR, a theory for which some version of SEP holds. Nonetheless, even for these equations it is true that they recover their special relativistic form in neighbourhoods of every point in which Riemann tensor terms can be ignored. This condition can be called approximate minimal coupling and it is this what I claim to be equivalent to the form of SEP that is taken as equivalent to the statement of the miracles. It is obvious that the same effect can be achieved by the condition of minimal coupling simpliciter, but this would be unnecessarily strong. These claims also assume that the matter fields do only couple to the metric and its derivatives, usually referred as universal coupling. Whether it is legitimate to use the term minimal coupling to what this version of the DA takes as primitive is mainly a question of terminology and not essential for the core argument of the paper. The essential question is, whatever the principle, whether there is one justification for it or it is taken as a brute fact. I thank an anonymous reviewer for pressing me for clarifying this point. 
plicit is to take as primitive the local validity of Poincaré invariance of all non-gravitational laws. The key point is that for the defender of the existence of the miracles in GR, the three versions seem the same claim under different names. Why this principle? Why this particular coupling? Why such symmetries? Nonetheless, the different versions can help to make explicit the relative strength of the claim, rooted in regarding its content as merely contingent in $\mathrm{GR}$, in relation to the weakness of the alleged explanation given in the GA, that seems committed to see it as necessary ${ }^{12}$ In any case, as I will argue below, seeing these formulations as equivalent also encapsulates the weakness of this version of the DA relative to other possible interpretations of what there appears as an unexplainable miracle.

I summarise here one version of the argument in support of seeing the miracles as unavoidable in GR. The miracles basically say that all non-gravitational laws are locally Poincaré invariant and that this invariance coincides, in a suitable neighbourhood, with the symmetries of the metric. Although this can be derived from some version of minimal coupling, it seems obvious that minimal coupling is not compulsory in GR. And even if from the DA perspective the metric earns the chronogeometrical significance by assuming SEP, understood as EP1', the principle is nothing more than a restatement of the miracle claims. So the miracles view is linked to the DA and a particular way of understanding SEP. It relies on approximate minimal coupling to the same metric not being forced a priori. And, finally, the argument also depends on there being no alternative way of explaining away the contingent coincidence of the symmetries of all non-gravitational laws and metric.

For the presentation of the logic behind the miracles claims to be complete, we must look at the places in which it is argued that other possible explanations of the alleged brute facts are doomed to fail. The main argument tries to show that the GA, even conceding that it had a story about how the matter laws survey the geometry of the primitive metric, cannot provide an explanation of the symmetries of laws that respects the contingency of the coupling. The argument thus focuses on showing through counterexamples that the coupling is in fact contingent in GR while necessary according to the GA ${ }^{13}$ If local Poincare invariance of the metric in the neighbourhood of any $p \in M$ explains the symmetries of the non-gravitational laws, then any law should reflect such symmetries. Read et al. present two scenarios (Jacobson Mattingly theory and bi-metric theories) which seem uncomfortable for the GA. Either if the approach argues that the metric constrains through some form of the relativity principle or in any other way, the counterexamples show that the reflection of the symmetries of the metric by the symmetries of laws,

\footnotetext{
12 An anonymous reviewer objects my use of modal terms, like contingent, to refer the fact that there can be theories for which some metric field might not have chronogemetric significance, matter laws might not have all the same symmetries or these might not coincide with the symmetries of the metric. Even if I agree that issues about modality might obscure the discussion, I find this an economical way to synthesise this idea. Necessary and contingent in this paper should be understood in this way, as referring to putative existence of theories that do or do not have those particular features.

13 [40, Section 6.
} 
found in GR, cannot hold of necessity ${ }^{14}$ In 39 it is argued that the version of the GA that could claim to explain the miracles is unviable for the reasons just mentioned and that a defensible version of the GA (there termed qualified geometrical approach (QGA)) needs to assume the miracles. In any case, if the only alternative to the DA is the GA, there is no satisfactory explanation for them.

Accepting that the GA cannot explain the miracles, we would need at least plausibility arguments to make the unlikelihood of finding other kind of explanations, not belonging to the GA, convincing. In 40, after recognising that an explanation of minimal coupling or some forms of the relativity principle would suffice to account for the miracles, it is claimed that no such explanation seems forthcoming, at least within the domain of GR. In [38] it is argued that in perturbative string theory, in contrast to what happens in GR, such an explanation can be found. Both the forecast and the argument will be questioned by the sections to come.

\section{What the miracles arguments take for granted}

In order to make explicit their assumptions, I present next a stylised version of the arguments presupposed in the claims of the existence of these unexplained brute facts in the domain of GR. This will provide a clear view of the interpretive commitments of the DA and will allow me, later, to consider what is involved in questioning them.

\subsection{DA: Miracles as an expression of SEP}

The first argument can be presented as follows: derive MR1 and MR2 from approximate minimal coupling and claim that such a derivation is non-explanatory as nothing forbids other kind of couplings between metric and non-gravitational fields. Alternatively, one could start from some version of the equivalence principle and note that it implies the miracles. Ironically, [40] note that in fact some forms of SEP are incompatible with some versions of minimal coupling, which indicates that the equivalence principle can be seen as constraining the couplings between non-gravitational fields and metric. This means that the argument assumes a version of the equivalence principle which is ineffective as a constraint. More specifically, it assumes a version of the equivalence principle (EP1') whose content is identical either to approximate minimal coupling or to the miracles, thus being explanatory idle. Let us look at the question of the formulation of the equivalence principle implied in this argument, and in general in the DA, in more detail.

As I discussed above, the DA distinguishes itself from the GA, in the context of GR, by claiming that the metric acquires chronogeometric significance through SEP understood as a codification of the miracles claim, that is, of

14 In 4.2 I will discuss some subtleties involved in these arguments. 
the claim about the coincidence of symmetries of matter laws and spacetime symmetries locally. This receives a concrete formulation in [40] through the claim of the validity of a particular version of the equivalence principle (EP1') together with the realisation that, due to the fact that the metric field in GR is locally Poincaré invariant, the symmetries of the metric and those of the dynamical laws coincide, implying that rods and clocks built out of such dynamical fields will survey the metric. Note again that the miracles are derived here from a version of the equivalence principle but, and this is what motivates referring to them as miracles, this particular version of the principle (EP1') is taken as nothing more than a reformulation of the validity of approximate minimal coupling. The question is whether there is another way of interpreting the equivalence principle such that it does not make the claims about the symmetries of the non-gravitational equations "miraculous".

It is informative to look at what Knox [26] calls the effective strong equivalence principle. Knox identifies empirical geometry with inertial structure defined operationally as those frames in which force-free bodies move with constant velocity and in which all the laws of physics take the same form 15 This notion of inertial frame would be then sufficient to determine effective spacetime structure ${ }^{16}$ But, as it is obvious from the definition, for it to be operative, we need a criterion to detect force-free bodies and the default form of laws. This is what the equivalence principle is supposed to do in GR, although in an indirect way: instead of using the notion of force-free body, the inertial structure is determined as the default structure detected by matter dynamics, were it shielded from all other interactions apart from the universal ones (if there are some) ${ }^{17}$ In fact, what the equivalence principle does in GR, in Knox's formulation of the effective EP, is to ensure that the operationally defined inertial frames coincide with the metric and connection normal frames. The precise formulation is the following:

Effective Strong Equivalence Principle (ESEP): The ESEP holds relative to some theoretical regime just in case, to any degree of approximation appropriate to the regime, given a sufficiently small region of spacetime, it is possible to find a reference frame with respect to whose associated coordinates the metric field takes Minkowskian form, and the connection and its derivatives do not appear in any of the equations of matter relevant to the regime 18

So, as Knox expresses it, SEP's job is to connect non-gravitational systems to the metric and connection (that somehow represent the gravitational field

\footnotetext{
15 See [26], p. 348.

16 According to Read and Menon's distinction 41 mentioned above this would be theoretical (not operational) spacetime.

17 This can be understood as an idealisation of the notion of force free-body (a body for which interactions are turned off) which incorporates, first, the possibility of universal interactions like gravity and also a field theoretical formulation. Taking this into account, one could say that the equivalence principle provides the determination of inertial structure as that detected by the matter dynamics when it does not interact with other matter.

18 [26], p. 353
} 
in GR) 19 For the discussion of the relation between the principle and the miracles, what is relevant is that Knox ends up giving a characterisation of EP that reduces it to something like approximate minimal coupling and that avoids, intentionally, explicit reference to gravitational interaction ${ }^{20}$ It is not difficult to see that Knox' ESEP is equivalent to what we have introduced, following [40, as EP1'.

Taking for granted that there is an open question about what the right formulation of the equivalence principle is, my claim so far is that the inevitability of the miracles, for the DA, is linked to a particular interpretation of this principle. The usual formulations of SEP seem to make the principle equivalent to the statement of the miracles. In Knox's formulation: that inertial frames, as determined by the non-gravitational laws, coincide with locally normal frames defined on the metric field ${ }^{21}$ is another expression of the miracles of general relativity. Then the principle of equivalence, that according to the DA is the key for the metric to acquire its chronogeometrical significance, is reduced to a mere statement of a matter of fact. The moral to extract from this part of the discussion is, then, that the miracles of general relativity, in the context of the DA, can be seen as a consequence of one particular tradition of understanding the equivalence principle which does not explicitly mention gravitational interaction. I will investigate below whether other interpretations of the principle can help to dissolve the miracles.

Furthermore, there is a remarkable feature in the formulation of the second miracle that is worth stressing as it provides a clue about how it might be explained. MR2 expresses what is supposed to be a brute fact about the coupling between non-gravitational fields and metric. As it was stressed above, for the statement to be valid in GR, the formulation must contain a restriction to suitable neighbourhoods which is dependent on the precision of the available measuring apparatuses. This is also apparent from the formulation of the effective equivalence principle (EP1' and ESEP) and tells us something about the kind of matter of fact that the miracle is expressing: it is not just a fundamental fact or property of the fields involved, it is a claim about certain features that are accessible at a given regime dependant on our apparatuses. This means therefore that the spacetime structure that is being surveyed, according to the miracles, is somehow effective or non-fundamental and that the principles, even if they are understood as statements of matters of facts, have an operational component. As I will claim at the end of the paper, this un-

\footnotetext{
19 In section [5], dedicated to the interpretation of the principle, I will expand on the connection between the motivating idea of the equivalence principle and GR's implementation.

20 I will argue in section 5 that this is a mistake: it is precisely the link to the original reference to free bodies what gives physical content to EP. This translates in the idea of inertial frames being determined through gravitational field that can be found, arguably, in some versions of the principle that we will meet below.

21 Knox introduces the notion of locally normal frames as a localisation of the notion of normal and orthonormal frames defined in SR (see 26], p. 349 for details). The aim is to have a characterisation for frames that can play the role of inertial frames in GR. The basic idea is that for locally normal frames on a geodesic one can find a neighbourhood where the connection coefficients vanish and the metric takes the Minkowskian form.
} 
avoidable element in the formulation of the miracles contains the key for their dissolution.

4.2 GA's failure to explain properly the constraints imposed by the metric

The second argument is concerned with the possibility of explaining the miracles in the GA. The explanation would be something like the following: the metric, an autonomous entity representing spacetime, somehow imposes constraints such that they force non-gravitational fields to approximately couple minimally (and universally) to the metric. If this were the case, it would imply that minimal coupling, and therefore the local Poincaré invariance of nongravitational fields, are true out of necessity. But, as the existence of different counterexamples show, this cannot be true.

The counterexamples presented in [40] are two. The first one is given by an action for an Einstein-Maxwell system with an added condition for the vector field that breaks the local Poincaré invariance: the Jacobson-Mattingly theory ${ }^{22}$ The reason for this to be a counterexample is that 'the dynamical behaviour of non-gravitational fields does not reflect the local (Poincaré) symmetries of the metric field - taken to represent spacetime." ${ }^{23}$ The second counterexample is provided by TeVeS bimetric theory ${ }^{24}$ In such a theory two metric fields can be defined: one of them is defined from the other plus a scalar and a vector field, which are also determined through field equations. The nongravitational fields minimally couple to the so-called physical metric as this is the metric surveyed by rods and clocks, light rays and whose geodesics test free bodies follow. The problem for the standard GA here is that the metric that has chronogeometrical significance in this theory is not the fundamental one, even though both are locally Poincaré invariant.

I will look at these kind of counterexamples in more detail when I discuss my alternative to the miracles in GR. Nonetheless, this initial presentation is enough to make explicit in what sense they are a problem for an eventual explanation of the miracles in the GA. They show that in some relativistic theories there can be a mismatch between local symmetries of (some of) the metric fields and the symmetries of the non-gravitational dynamical laws or that an expression like the matter fields survey the metric might not be well defined. The problem for the GA comes from assuming that the spatio-temporal character of the metric is given a priori and that any constraint for the coupling of non-gravitational fields to the metric comes from the direction of the metric and implies some kind of necessity. This without counting the problem of not specifying the nature of such constraints.

Let me finish this section by pointing out that what have been presented as the main problems for explaining the miracles in the two approaches are potential elements for their dissolution. The DA regards SEP, in the form

22 See 23

23 40, p. 8

24 See Bekenstein's paper [3] for the original formulation of the theory. 
of EP1', as giving chronogeometrical significance to the metric but trivialises SEP and makes it equivalent to approximate minimal coupling: this is why assuming SEP is seen as equivalent to stating a collection of brute facts. The GA implicitly claims that constraints coming from the metric would explain the miracles insofar as matter is forced to survey the spacetime metric. But either it considers the geometrical meaning of the metric as primitive and then it is not understandable how the constraints work and why they are not necessary or, in a qualified version that takes out the metaphysical meaning from the metric, assumes the constraints also as brute facts. ${ }^{25}$ In any case, both GA and DA are insufficient to explain the miracles, which does not mean that the equivalence principle and the idea of the determination of the metric imposing certain constraints on the behaviour of matter fields are useless as clues in the search for an explanation.

\section{How to account for the miracles}

After considering the logic and basic assumptions of the miracle claims, we have the following situation. The defining claim of this position is that there are some features in the foundations of relativity, expressed by MR1 and MR2, that are not only surprising or puzzling in the theory but whose explanation in the theory does not seem possible ${ }^{26}$ Furthermore, the features in question are taken to be a necessary component of the requisites for the metric to have its chronogeometrical, and therefore, spatio-temporal, character ${ }^{27}$ The miraculous nature of such features is defended primarily as a consequence of the couplings between metric and non-gravitational fields not being determined a priori in GR: something that must be assumed both by the GA and the DA. So it must be taken as an unexplainable brute fact. Any eventual explanation of the miracles must then account for the contingent character of the chronogeometry of the metric while, at the same time, introducing some kind of explanatory nomicity. My claim is that certain way of interpreting some fundamental principles of the theory can achieve exactly this. In the rest of this section I present the elements of such an explanation.

\footnotetext{
25 See 38 for discussion of different versions of the GA.

26 Expressions to this effect can be found, for instance, in [40] and [39].

27 This assuming that, as it seems to be assumed in this version of the DA, there is no other way of accounting for the spatio-temporal character of the metric in which the miracles are not presupposed. The Ehlers-Pirani-Schild construction ([16]) and the proposal developed in the rest of this paper can be taken as attempts at proving this posit wrong. Read ([39], footnote 55) acknowledges the first possibility, although he argues that it does not provide a complete explanation of the miracles.
} 
5.1 The miracles as derived from a principle

In [40] it is suggested that an explanation of minimal coupling or certain forms of a relativity principle could account for the miracles but that "no such explanation appears to be forthcoming" 28

My general claim is that GR provides the tools to account for the miracles or, in other words, that the coincidence of all the local symmetries of the non-gravitational field equations and of these with the local symmetries of the metric, in certain regimes, is not as miraculous in GR as it is claimed. The first part of my claim is that the equivalence principle, properly understood, provides the framework to account for the miracles. The obvious question then is about how to understand the principle so that it can play that role. The answer, I advance, is this: As a principle that prescribes the determination of inertial frames through the gravitational interaction. This interpretation of the principle exploits the fact, to be developed below, that in GR, the Einstein field equation, in a sense the equation that governs the gravitational interaction, can be seen as imposing some constraints on the matter that sources such interaction. Such constraints are what the miracle claims are expression of. In what follows I will expand on this.

In previous sections $3,4.1$ we have encountered different versions of the equivalence principle. I have defended there that one thing that they have in common is that they can be taken as a reformulation of the miracles. This has the consequence, according to the above mentioned maxim of the DA (that the metric earns chronogeometric significance by way of SEP), of regarding the spatiotemporal character of the metric in GR as fully unexplained ${ }^{29}$ There is something strange here, as it is odd to interpret a principle in a way that makes it equivalent to a mere statement of a matter of fact. Even recognising that there is a factual component in any operative principle, it is clear that there must be more involved in its formulation than an expression of a matter of fact to deserve such name. In any case, irrespective of one's position in relation to the general status of principles, I claim that the equivalence principle in GR has more content than the re-statement of the miracle claims.

To see this, recall two historical formulations of the principle of equivalence. They might be incomplete and differ in important respects from the versions of SEP presented above, but it is relevant to bring them up here as they point in the right direction if one wants to determine the physical content of the principle. Not in vain do both of them make an explicit reference to gravity. The first is Einstein's formulation of 1918:

\footnotetext{
28 40, Section 8.

29 Claims like this one assume a general operational perspective under which the spatiotemporal character of the metric is acquired through the physical behaviour of rods and clocks made out of matter fields and a formalist concretion of the functionalist intuition (of which 26] is an example) to the effect that such behaviour of rods and clocks will be encoded on the symmetries of the equations of motion of matter fields. If something like this is assumed, the miracle claims would regard the spatiotemporal character of spacetime as a brute unexplained feature in the DA. For more on the relation between the operational perspective of the DA and its relations to Knox' spacetime functionalism see [4]
} 
Principle of Equivalence. Inertia and gravity are phenomena identical in nature. From this and from the special theory of relativity it follows necessarily that the symmetric "fundamental tensor" determines the metric properties of space, the inertial behaviour of bodies in this space, as well as the gravitational effects. We shall call the state of space which is described by this fundamental tensor the "G-field." [14

Pauli, a few years later, writes

For every infinitely small world region (i.e. a world region which is so small that the space and time variation of gravity can be neglected in it), there always exists a coordinate system $K_{0}\left(X_{1}, X_{2}, X_{3}, X_{4}\right)$ in which gravitation has no influence either on the motion of particles or on any other physical processes. ([35], p. 145).

These two formulations of the principle are very different; the first one being closer to what is usually called Weak Equivalence Principle and the second to SEP. If I bring them together here it is because both make explicit reference to the role of gravity in the determination of inertia ${ }^{30}$ Pauli's version suggests that whatever gravity is, there is always a region of spacetime in which its effects on the motion of particles or other physical processes can be neglected. Einstein's formulation identifies gravity with inertia and from this extracts the conclusion of both phenomena being represented by the same object, the metric field, that is no other than the field determined through Einstein's field equation.

The use of the concept of gravitation in the formulation of the equivalence principle has been objected to, mainly on the basis that it is ambiguous what this term means in GR and even questionable that it makes any sense in it 31 Nevertheless, the complete specification of its referent is not a necessary condition for the principle to make sense. In GR, we have field equations that govern gravitational interactions. The use of "gravitation" in the expressions of the equivalence principle can be understood as referring to whatever is determined by such equations, irrespective of whether this plays exactly the same role as the gravitational field does in Newtonian gravity. The relevant question here is whether we can make use of the concept of gravitation in a way that is operative to formulate a principle of equivalence. My previous claim is that without mentioning the phenomenon of gravitation in its formulation, the principle loses part of its physical content.

\footnotetext{
30 An anonymous reviewer points out that my use of the formula "gravity determining inertia" is highly questionable when applied to Einstein's version of the principle, as he would not concede a fundamental status to any of the concepts involved. Even agreeing with this historical remark, and being aware of the differences between the two principles here presented, I still think that it is illustrative to introduce them here as examples of the explicit reference to gravity in the formulation of the principle, something that will be exploited in my use of the equivalence principle as part of the explanation of the miracles. 31 See 26. For a discussion on whether the metric field can be understood as a gravitational field in GR and a characterisation of geometrical and gravitational significance for mathematical objects, see 27. According to Lehmkuhl's analysis, it is possible to maintain that the metric has both gravitational and geometrical significance.
} 
So if the versions of the principle by Pauli and Einstein are objectionable it is not because of their direct reference to gravitation but because of certain imprecision in their formulation that should be corrected. The guiding idea to do so is the following: the local inertial frames detected by matter dynamics, and in this sense the effective spacetime structure, are determined by gravitational interaction. One first presicification for this principle is the following: the local symmetries of non-gravitational fields and motion of force-free bodies (i.e. local inertial frames) are determined, in a neighbourhood to a good degree of approximation, by the gravitational interaction (through Einstein's field equation or the corresponding gravitational field equations) 32

As I will argue below, in GR, the Einstein field equation determines the values of the gravitational interaction while constraining, approximately, the local symmetries of non-gravitational field equations and the motion of test bodies. This is the main sense in which the equivalence principle is implemented in GR.

\subsection{How the gravitational field equations constrain matter laws}

As we have seen 4.2 , the GA attempts to account for the miracles by claiming that the metric field somehow constrains the non-gravitational field equations. But the problem is that it does it badly: it does not explicitly say what the constraints consist in and it implies that they hold out of necessity. Nevertheless things can be presented differently. I will show that in GR, the Einstein field equation constrains the equations for the non-gravitational fields by imposing that, insofar as such fields are sources for the gravitational fields, such equations must be locally Poincaré invariant, and the motion of force-free bodies is approximately geodesic 33 This provides content to the attempts at explaining the constraints of the non-gravitational laws by the metric, carried out in the $\mathrm{GA}$, in a way that it does not presuppose any mysterious a priori connection and that is relevant to accounting for the miracles. In this section I develop the logic behind such constraints.

The dynamical equations for the gravitational field in GR, given by Einstein's field equation, relate the metric and its derivatives to the energy-

\footnotetext{
32 This is only a first expression of the principle. At this point, without further explanation, it can be seen as not substantially different from claims identifiable with some version of the GA (for instance, Read's QGA presented in 39]) indicating that some field, the metric field, constrains the couplings of all the non-gravitational fields. This is right insofar as any explanation that intends to elude the miracles must refer to some common origin of the constraints, but it is not the full story. The rest of the story comes from making explicit the physical content of the principle in GR (and other theories) as implemented by the gravitational field equations. See below for further discussion.

33 Geodesic motion of test bodies can be derived from the conservation equation under certain conditions through different geodesic theorems (the Geroch-Jang being one of them). For test bodies made out of the conserved matter fields the results would establish that its worldtube contains a timelike geodesic. See [20, 12, [21, 46], 47]. More on the relevance of geodesic theorems for the explanation of the miracles below.
} 
momentum distribution encoded by the energy-momentum tensor for nongravitational fields.

$$
G_{a b}:=R_{a b}-\frac{1}{2} g_{a b} R=8 \pi T_{a b}
$$

For later reference, let me note that the Einstein field equation can be derived from Hamilton's principle by extremising the Einstein-Hilbert action, plus an action defined for the matter fields, with respect to $g_{a b}$.

$$
\begin{aligned}
S=S_{G}+S_{M} & \\
S_{G} & =\int R \sqrt{-g} d V \\
S_{M} & =\int \mathcal{L}_{M}\left(g_{a b}, \phi^{i}\right) d V
\end{aligned}
$$

$\mathcal{L}_{M}$ is the Lagrangian density for the non-gravitational matter fields, $\phi^{i}$, where $i$ is an index denoting a specific matter field. The equations of motion for the matter fields can be derived by extremising $S_{M}$ with respect to these non-gravitational fields. In the Lagrangian formulation, the energy-momentum tensor is defined by $T^{a b}:=\frac{\delta \mathcal{L}_{M}}{\delta g_{a b}}$.

Given this, in GR, there are two paths to deriving and justifying the conservation of the energy-momentum tensor field $\left(\nabla_{b} T^{a b}=0\right)$. The first starts from Einstein's field equation and derives the equality making use of Bianchi identities (mathematical identities that guarantee the vanishing covariant divergence of Einstein tensor with respect to the derivative operator compatible with the metric: $\left.\nabla_{b} G^{a b}=0\right)$. The second consists in deriving it as a consequence, partly, of the invariance properties of the matter action. To be more specific, the conservation of $T^{a b}$ with respect to $\nabla$ can be derived from the invariance of the action under the pushforwards of the metric and non-gravitational fields induced by diffeomorphisms, together with the assumption that the Lagrangian density $\mathcal{L}_{M}$ depends only on the metric compatible with $\nabla$, the matter fields and their covariant derivatives; and assuming that the equations of motion for the non-gravitational fields are satisfied ${ }^{34}$ This is usually formulated in terms of the conservation condition being derived from the diffeomorphism invariance of the matter action but, as it is stressed by Weatherall, the relevant point in this inference is that it determines that the derivative operator with respect to which $T^{a b}$ is conserved is the one compatible with the same metric that is varied in the definition of the tensor and that enters in the equations of motion of the matter fields; the metric, therefore, to which matter fields (minimally) couple.

The relevance of having these two ways of deriving the conservation condition has received some attention in relation to the discussion about the status of the geodesic principle in GR ${ }^{35}$ Brown originally claimed that the availability

\footnotetext{
34 See 49 for a detailed derivation of this result.

35 See [6], 29], 37], 49], 48], 42]
} 
of the first path, given the existence of some results that connect the conservation condition to the geodesic motion of test bodies, proves that inertial motion is a theorem, rather than an assumption, in GR. The claim has been tempered in several respects: by noting that the derivation of geodesic motion, encoded in the so-called Geroch-Jang theorem, requires also satisfaction of the strengthened dominant energy condition (SDEC), not a consequence of the Einstein field equation, and that it is, in fact, independent of whether the metric is a solution of the equations; by stressing that this means that the result might be also derived, due to the existence of the second route to obtain the conservation condition, in theories with fixed spacetime metric like SR; and by showing that some analogous result can be derived in geometrized Newtonian gravity. All this, no doubt, must be taken into account to moderate the initial claim ${ }^{36}$ Nevertheless, the fact that we have these two routes and, in particular, the availability of the first one in GR, but perhaps not in other theories, is essential for the discussion of the miracle claims.

The reason behind the last point is the following. In GR it is possible to derive the condition $\nabla_{b} T^{a b}=0$ from Einstein's field equation, i.e. from the dynamical equations for the gravitational interaction. From this equality, under the conditions needed to prove the Geroch-Jang theorem ${ }^{37}$, one can derive the (approximate) geodesic motion of force-free bodies and, as I will argue below, local Poincaré invariance. And, as we have seen, these are the features that, according for instance to Knox, characterise effective spacetime through the notion of local inertial frame. What this means is that the Einstein field equation can be seen as constraining the dynamics of the matter (nongravitational fields), that acts as a source for the equation, to survey this effective spacetime structure. Such a constraint is what gives content to the equivalence principle in a way that can explain MR1 and MR2.

Before unfolding the last point, let me justify the claim that from the vanishing covariant divergence of the energy-momentum tensor one can derive the local Poincaré invariance of the equations for the non-gravitational fields. Read in 39 claims that proving something like this requires assuming what is called the Schiff's conjecture. But, as he notes ${ }^{38}$ Schiff's conjecture states that a theory satisfying the weak equivalence principle would satisfy also the strong one or, similarly, that from geodesic motion we could derive local Poincaré invariance. What we need here is something weaker; it is to derive local Poincaré invariance for all matter laws that can be associated individually to a energymomentum tensor conserved with respect to a derivative operator compatible with the same metric that defines the Einstein tensor (recall that this is what is derivable from Einstein's field equation). The claim, then, can be taken in this weak conditional version: if the $T^{a b}$ tensor is to be associated with the energy-momentum of some non-interacting non-gravitational fields which are determined by some dynamical equations, then the equations governing those

\footnotetext{
36 For discussion on the relevance of these amendments, see [29, 48, 42.

37 These are basically, the strengthen dominant energy condition and the small-body limit. See [29], 48] for details.

38 39.
} 
non-gravitational fields are at most locally Poincaré invariant ${ }^{39}$ The reason is that if the fields in question are associated with an energy-momentum tensor that is divergence-free with respect to a metric (one that can be part of a relativistic spacetime $\left.\left(M, g_{a b}\right)\right)$, then as it is always possible to define a locally normal frame, that is, a tetrad field that is normal on the geodesics of that metric, this means that the connection coefficients with respect to that frame vanish on that curve and that the metric takes approximately the form of the Minkowski metric in a small neighbourhood $N$ of the geodesic $\left(\left.g_{a b}\right|_{N}=\eta_{a b}\right)$ On such a neighbourhood $N$, as I argue below, from $\nabla_{b} T^{a b}=0$ we can derive (approximately) $\partial_{a} T^{a b} \omega_{b}=0$ (being $\omega_{b}$ a Killing vector field of the Minkowski metric). And if $T^{a b}$ is the rank-two tensor representing the energy momentum of matter fields for which $\partial_{a} T^{a b} \omega_{b}=0$, the equations for which such fields are solutions must be Poincaré invariant in the neighbourhood $N$.

Let me spell out the steps that lead to this conclusion. For any symmetric divergence free tensor field, like the energy-momentum tenor $T^{a b}$, and a Killing field $\omega_{b}$ with respect to $g_{a b}$, it follows that $\nabla_{a} T^{a b} \omega_{b}=0 .{ }^{41}$ Now take a locally normal frame as defined above on a point $p$ : it is the case that in that frame [at $p$ ] the metric takes the Minkowski form and the connection coefficients vanish. There is always a sufficiently small neighbourhood of $p$, the empirical determination of which will depend on the precision of the available measuring apparatuses, in which the metric is approximately Minkowskian up to a given order. It is possible, then, to choose such a neighbourhood in a manner that the connection coefficients vanish in the locally normal frame $\left(\left.\Gamma^{n}{ }_{m l}\right|_{N}=0\right)$. Hence, using the ten Killing vector fields of the Minkowski metric, we can define quantities $T^{a b} \omega_{b}$ that, in the locally normal frames defined (and approximately on any sufficiently small neighbourhood of any $p \in M$ ), meet the condition $\partial_{a} T^{a b} \omega_{b}=0$. Note that here I have made use of the fact that the $\omega_{a}$ are Killing fields of $\eta_{a b}$ and the connection coefficients vanish, which can be only approximately assumed. Nonetheless, under such assumptions, we have derived ten ordinarily conserved currents (only locally approximately valid)

\footnotetext{
39 The qualification here is due to the fact that one could restrict further the symmetries of matter laws through the introduction of fixed fields that break local Poincaré invariance. This being so, the question relevant for the present approach is whether this is a problem for the claim of the miracles being explained in GR. The answer is that it is not so: a theory thus modified would not contain an explanation of the miracles but it would neither satisfy the version of the equivalence principle put forward in this paper. If we had a Lagrangian formulation for it, we would need a term in which the fixed field couples to the matter fields. This would violate the version of SEP that I present here as an explanation of the miracles, as it would not be true that just the gravitational field equations would determine the local inertial frames. One might think that the fixed field could perhaps be dynamically determined through equations that one might want to call gravitational; if this were the case, it would satisfy the principle as formulated here and allegedly explain the miracles, but the theory, if consistent, would not be GR anymore.

40 See [26] for details on the definition of locally normal frames. See [17] for a precise characterisation of the notion of approximate local spacetime symmetry.

41 A Killing field $\lambda$ with respect a metric $g_{a b}$ is defined as a smooth vector field for which the Lie derivative of $g_{a b}$ associated with it vanishes: $£_{\lambda} g_{a b}=0$. The (local) flow maps determined by the Killing fields are isometries of the metric. See, for instance, [30], p. 75
} 
from the covariant conservation of the energy-momentum tensor $\left(\nabla_{b} T^{a b}=0\right)$ derivable form the Einstein field equation.

The next step would be to infer the invariance properties of the nongravitational field equations from these conserved currents. One can do so at least for equations that are derivable form an action through Hamilton's principle. In such theories, one can derive the transformation properties of an action integral from the existence of divergences of some quantities by applying the converse of Noether's first theorem ${ }^{42}$ This is how it would work out in this case. Let us take the matter part of the action in equation 2 and calculate the variation under the one-parameter diffeomorphisms generated by an arbitrary vector field $\omega_{a}$. It follows that

$$
\delta S_{M}=\int \delta \mathcal{L}_{M}\left(g_{a b}, \phi^{i}\right) d V=\int\left(\frac{\delta \mathcal{L}}{\delta g_{a b}}\right) \delta g_{a b} d V+\int\left(\frac{\delta \mathcal{L}}{\delta \phi_{i}}\right) \delta \phi_{i} d V
$$

The last term of the second expression vanishes just assuming that the equations of motion for the non-gravitational field are satisfied. Substituting the definition of the energy-momentum tensor, we obtain

$$
\delta S_{M}=\int T^{a b} \delta g_{a b} d V
$$

where the variations of the metric, in this case, can be written as its Lie derivative associated with the vector field that generates the transformation, $\delta g_{a b}=£_{\omega} g_{a b}=\nabla_{(a} \omega_{b)}$. Substituting in 4 , we obtain

$$
\delta S_{M}=\int T^{a b} \nabla_{(a} \omega_{b)} d V=\int \nabla_{a}\left(T^{a b} \omega_{b}\right) d V-\int\left(\nabla_{a} T^{a b}\right) \omega_{b} d V
$$

Now, the second term of equation 5 is the conservation condition derived from the Einstein field equation that vanishes identically (as said above, it is also derivable from the diffeomorphism invariance of the matter action) and, interestingly, the first term also vanishes if we take $\omega$ to be a Killing field, something that we can only do under the provisos made above for the locally normal frames. It must be stressed that such a term cannot generally be neglected as we have not assumed that the transformations vanish on the boundary; it can only be so neglected in the case of $\omega_{a}$ being a Killing field with respect to the metric, something that it is only locally approximately true here. The result, then, is the local invariance of the action under the

\footnotetext{
42 In her 1918 seminal paper 33, Noether proved two theorems and their converses. The general result connects the transformations of the dependent and independent variables that are invariances of an action integral with certain combinations of the Euler-Lagrange expressions. From this, she derives the two theorems: the first applies to finite groups of transformations (transformations that depend on constant parameters) and derive that certain combinations of the Euler-Lagrange expressions are divergences; assuming that such equations are satisfied, the divergences vanish and we obtain the conserved currents. The second, for transformations depending on arbitrary functions, provides further identities between the Euler-Lagrange expressions. Noether also proves the converse of these two theorems. The one that is of interest here is the converse of the first one, that would allow derivation of invariances of the action from the ten conserved currents that we have.
} 
transformations generated by the ten Killing vector fields of the Minkowski metric 43

The final step is provided by the following result: if the action is invariant (up to a divergence term) under certain transformations, then the equations of motion derived from it through Hamilton's principle are form invariant under such transformations ${ }^{44}$ Then, from the conservation expressions $\partial_{b} T^{a b}=0$, we have proved the existence of equations of motion that are Poincaré invariant. As this holds only on certain neighbourhoods of each point, the claim has only local approximate validity. This translates in the following claim: any matter field that can act as a source in the Einstein field equation (and whose field equations can be derived from an action principle) is such that satisfies locally Poincaré invariant field equations. The gravitational interaction, as described by GR, constrains local symmetries of all matter laws to be the same (Poincaré) and to coincide with the local symmetries of the metric 45

My claim is that this is sufficient to explain the miracles, as I will defend below, for matter that can act as source of the gravitational field equations.

\subsection{Miracles explained}

I first summarise what has been achieved so far. In the previous section, I have shown in what sense we can say that the satisfaction of the Einstein field equation imposes constraints on the dynamics of non-gravitational fields: the possibility of deriving the conservation of the energy-momentum tensor from the equations for the gravitational field, and from it approximate geodesic motion and local Poincaré invariance of laws, supports the claim that the validity of Einstein's field equation somehow constrains matter behaviour, even recognising that the conservation condition can be derived independently; by assuming, for instance, that the equations of motion for the non-gravitational fields are derivable from a Lagrangian density meeting some general conditions (more on this below).

This has several consequences. First, it tempers some claims to the effect that GR allows any coupling of the non-gravitational fields to the metric whatsoever. It does so insofar as either the couplings in question respect the constraints or they are non-gravitational fields that somehow do not contribute to the energy-momentum tensor that is placed on the righthand side of Einstein's field equation. Second, the content of the constraints is precisely what [40] present as the miracles of relativity: that all the non-gravitational interactions (that act as sources in the Einstein field equation) are locally governed

\footnotetext{
43 As an anonymous reviewer points out, this derivation of the invariance properties of the action presupposes that the equations of motion for the matter fields are satisfied. The reviewer wonders whether this is a legitimate move; my impression is that it is so insofar as we are interested in deriving constraints for equations of motion that can be derived from an action for which the defined energy-momentum tensor is conserved.

44 For this result, see [1], section $9.3 ; 4$.

45 It must be stressed here that the derivation that supports this claim presupposes the non-existence of fixed fields to which the matter fields couple. See my footnote 39
} 
by Poincaré invariant dynamical laws and that such symmetries coincide, in certain regimes, with the symmetries of the metric field. What is stated in the miracles is a consequence, according to this view, of the energy-momentum tensor being divergence-free with respect to a particular metric (and this can be seen, in GR, as a consequence of the Einstein field equation). Third, even if this corrects the claims made in the context of the DA in relation to the miraculous nature of certain features of non-gravitational interactions in relativity, in a way that I will fully specify next, it does not give comfort to the GA. It is true that there are constraints on the couplings between metric and non-gravitational fields, but they have nothing to do with a primitive geometrical nature of the metric. They are imposed, in GR, as a consequence of regarding the gravitational interaction as governed by certain fundamental dynamical equations together with an operational perspective on the understanding of emerging spacetime, which is understood as such because of the chronogeometrical significance acquired through the geodesic motion of forcefree bodies and the local Poincaré invariance of laws (which makes available a notion of local inertial frame). The constraints, then, should not be understood as being directed from spacetime to matter laws but rather as originating in the conjunctive determination of the gravitational interaction and the effective spacetime structure.

This has a direct impact on the DA perspective on the miracles and the question of the role of the equivalence principle in their eventual explanation. The two claims presented in the two previous subsections - that the equivalence principle properly understood provides the framework to account for the miracles and that Einstein's field equation constrains matter to behave as the miracles say - converge here to provide a plausible explanation of the miracles in GR. And they do so by providing also an interpretation of the equivalence principle in GR that makes it explanatory in this context. The equivalence principle is understood as a claim about determination of inertial frames through the gravitational interaction dynamically governed by field equations (the Einstein field equation). It contains a claim about the Poincaré invariance of non-gravitational laws in suitable neighbourhoods (basically the content of EP1') but together with the claim that this is a consequence of the dynamical equations that determine the gravitational interactions (in the case of GR, a consequence of the Einstein field equation). The first claim is the one that is normative and extrapolable to other theories: we can impose the same kind of couplings for theories different to GR; theories in which matter seems to survey the same relativistic spacetime even though the equations for the gravitational field(s) are different or nonexistent. I will discuss how to translate the discussion to these scenarios in the next section. Nevertheless, if one retains only this first claim, without reference to anything else, the principle is either unjustified (what forbids other couplings?) or the statement of a massive coincidence. The second claim can be understood as an empirical condition that must be met if the first claim is to hold: insofar as there is an interaction, similar to what is classically known as gravity, which is determined by certain equations from which local Poincaré invariance of non-gravitational 
laws (and approximate geodesic motion) can be derived, this fact can be used to define local inertial frames and endow the metric with its chronogeometrical meaning ${ }^{46}$ Not all theories have the resources to implement this part of the principle (obviously not non-gravitational ones).

The two roads presented above for the derivation of the conservation condition can be seen as corresponding to the two claims of the equivalence principle. The general claim about coupling (or conservation), which might be valid in a number of theories, is correlated to the fact that for any matter Lagrangian density that depends on a given Lorentzian metric, some matter fields and their derivatives, it is possible to derive the conservation condition (the second route). In principle form, the claim would imply that every matter Lagrangian density must respect these conditions. But then, questions arise as to why this must be so: what does the metric that appears there have to do with the one being dynamically determined through some equations (if this is the case) and, even more basically, why a Lorentzian metric in the first place: ${ }^{47}$ The main reply again is that, given this in isolation, either it is a principle for which we have no justification or we must assume that it is just a matter of fact.

The claim about gravity determining locally the inertial frames is implemented in GR by the first route for deriving the conservation condition. In other theories, if there is a way to derive the conservation condition (or any other from which local Poincaré invariance and approximate geodesic motion can be inferred), we will be able to say that a principle of equivalence similar to the one valid in GR obtains and, in this sense, that the miracles get explained in those theories. This means that this version of the principle provides a criterion to decide which theories are, in this sense, closer to GR 48 On the other hand, the different versions of SEP presented above, which only take into account the claim about local symmetries of laws divorced from the claim about the gravitational interaction, become either a factual claim about the couplings or an empty principle applicable to many theories, and are unable on their own to provide an account of the miracles and, therefore, of the chronogeometrical meaning of metric.

\footnotetext{
46 One might object here that talk of gravitational interaction in the context of GR, or relativistic theories in general, is not sufficiently clear for the claim to have a substantive content. I think that it is enough, for the purpose of this paper, the identification of the equivalence principle with the claim that objects with gravitational significance (in the sense defined by [27]) are determined by equations that might impose the constraints that provide chronogeometrical meaning to some of these objects.

47 In Section 7 I will have more to say about this last question.

48 For a condensed presentation of different metatheoretical frameworks proposed to compare GR to other spacetime theories, see [28].
} 


\section{Beyond GR: how this dissolution of miracles works for bimetric theories}

We must see how this approach on the miracles and the equivalence principle fares for theories that according to [40] are problem cases to the alleged explanation of the miracles by the GA.

As discussed in 4.2 these cases pose a problem for the alleged explanation of the miracles in the GA. If one wants to claim that the local Poincaré invariance of all non-gravitational laws is a consequence of them living in the same spacetime structure, then it seems that the symmetries of the metric, interpreted as spacetime structure, are necessarily imposed on the non-gravitational laws in GR (as in SR, the Poincaré invariance of matter laws would be a consequence of the fields being placed in a Minkowski spacetime). Leaving aside the question of how the geometry of spacetime imposes such constraints, it seems that theories for which the symmetries of the matter laws do not reflect locally the symmetries of the metric are problematic for this view.

Let me, nonetheless, insist that these cases are problematic for a view claiming that the metric has a primitive spatio-temporal meaning and that fields living in a given spacetime must all reflect the local symmetries of the spacetime metric. But not, as I will show, for the perspective defended here, in which the constraints are imposed by the equivalence principle. There are, at least, two potential types of difficult cases: theories for which the symmetries of the metric and of the non-gravitational laws do not coincide and theories with more than one metric.

As already discussed above, 40] present two theories as challenges to the explanation of the miracles in the GA. The version of the Jacobson-Mattingly theory discussed is one that belongs to the first type in the following sense: the equations of the theory are derived from an Einstein-Maxwell action with a term added that imposes, using a Lagrangian multiplier, that the vector potential be locally timelike. Here the problem is, as [40] note, that the 'dynamical behaviour of non-gravitational fields does not reflect the local (Poincaré) symmetries of the metric field - taken to represent spacetime."49 From the perspective presented in this paper, this is a case where further constraints, beyond the one coming from the gravitational field equations, are imposed. This is not forbidden by the equivalence principle, as considered here, but it might be seen as unjustified. In fact, in this Jacobon-Mattingly theory, it is a condition imposed by hand through a Lagrangian multiplier. If the vector field were a dynamical player, this would result in a modification of the energy-momentum tensor and therefore in the emergence of a spacetime structure surveyed by matter that might be different to the original fundamental metric. This is seen clearly in the second type of problematic theories.

Bekenstein's TeVeS theory is a prototypical case of a bi-metric theory that gives a hard time to a geometrical perspective. In such a theory, on top of the metric field and the non-gravitational fields, we have two other "gravita-

49 [40], p. 22 
tional" fields that satisfy their own field equations derivable, also, from the corresponding part of the action. Moreover, as mentioned above, the theory can be written as a bi-metric one: doing so makes clear that the metric that the non-gravitational fields survey is not the "fundamental" metric - the one used in the definition of Einstein's tensor and the part of the action that is imported from GR - but one that is defined from the fundamental metric plus the vector and scalar fields (the so-called physical metric $\tilde{g}_{a b}$ ). This is explicit in the form that the matter action takes: it can be written in terms of the $\tilde{g}_{a b}$, the non-gravitational fields and their derivatives with respect to the derivative operator compatible with $\tilde{g}_{a b} 50$

This theory is problematic from the perspective of the GA. Why matter fields do not survey the fundamental metric, or which metric has the chronogeometrical significance, are questions that the GA lacks the resources to answer. But, at the same time, the example is very illustrative of how an account of the chronogeometricity of the physical metric, and eventually of the miracles, might work. The defenders of the DA may see their claim about the miracles confirmed in this case, as one can defend that it is the local symmetries of non-gravitational laws that determines which metric is surveyed by rods and clocks; and what these non-gravitational laws be is, in the end, a matter of fact. Nonetheless, this story is incomplete. If one takes all the gravitational field equations together, they imply that the energy-momentum tensor for the non-gravitational fields be conserved with respect to the covariant derivative compatible with $\tilde{g}_{a b}$. We have then, analogously to GR, two paths to derive $\widetilde{\nabla}_{b} \widetilde{T}^{a b}=0$ : one is to derive it form the fact that $\mathcal{L}_{M}$ depends, in this case, on matter fields and $\tilde{g}_{a b}$ and apply the same procedure than for the matter Lagrangian in GR. The other one is to impose the field equations for all the gravitational fields and derive the conserved condition with respect to the derivative operator compatible with $g_{a b}$ this time. The remarkable thing, in this case, is that such condition can be written as $\widetilde{\nabla}_{b} \widetilde{T}^{a b}=0$ for some metric $\tilde{g}_{a b}$

So, what to make of this? Here we have a beautiful illustration of the two components of the equivalence principle. That the theory has a part of the action dependent only on the physical metric and matter fields is an expression of that component of the principle that claims that all matter laws share the same local symmetries between them and with a metric field. This is a

\footnotetext{
50 Apart from Bekenstein's original paper, 6] and 49] contain discussions of the status of the conservation condition in this theory.

51 This claim is based in consistency reasons: the theory has been designed for the matter fields to couple to a physical metric that is defined from the metric and these other gravitational fields, meaning that the results from the two paths must be compatible for the theory to be consistent. Direct calculation should reflect this fact.

52 In order to prove the conservation condition of the energy-momentum tensor with respect to the derivative operator compatible with $\tilde{g}_{a b}$, one must only note that the matter Lagrangian for this theory has the same form as the one in GR where $g_{a b}$ is replaced by $\tilde{g}_{a b}$ (for an explicit proof see [49]). To go from $\widetilde{\nabla}_{b} \widetilde{T}^{a b}=0$ to the local Poincaré invariance of non-gravitational laws, one must apply the same derivation presented above for the case of GR (section 5.2 ), taking into account that $\tilde{g}_{a b}$ is also a Lorentzian metric.
} 
miraculous coincidence if it is taken by itself; in fact, in TeVeS, it is imposed as a defining condition of the theory, as the proponent of the theory was searching to modify the general relativistic gravitational field equations changing the metric that is surveyed by matter fields in order to be able to account for certain cosmological phenomena. But the other perspective is also available: in this theory, the gravitational interaction, which is needed to determine inertial frames, is described differently to GR. From this perspective, the equivalence principle is still valid but changes the form of the gravitational interaction that provides its specific content. And in a similar way, we can say that we have an account for the two miracles and a comprehension of why they also hold here, but for the physical metric instead of holding for the fundamental one.

A third touchstone for a proposal like this one is provided by Brans-Dicke theory ${ }_{53}^{53}$ Brans and Dicke formulate a theory with two non-matter gravitational fields (the metric and a additional scalar field) with the aim of providing a theory that is more Machian than GR ${ }^{54}$ In order to obtain that matter freebodies move on the geodesics of the metric $g_{a b}$, they ensure that the matter fields in the Lagrangian coupled directly to the metric and only indirectly to the scalar field:

$$
L=\sqrt{-g}\left(\phi R-\frac{\omega}{\phi} g^{a b} \nabla_{a} \phi \nabla_{b} \phi+16 \pi L_{M}\right)
$$

Where $\omega$ is a dimensionless coupling constant, $\phi$ a masless scalar field and $L_{M}$ is the ordinary Langrangian for matter fields couple to $g_{a b}$.

By varying with respect to $g_{a b}$ and $\phi$ we obtain equations for the metric and the scalar fields. Note that here, if one imposes satisfaction of these equations (the new gravitational field equations) one obtains conservation of the energymomentum tensor and an explanation of the miracles similar to the one in GR. Note also that the conservation of the ordinary energy-momentum tensor with respect to the derivative operator compatible with $g_{a b}$ can be derived, like in GR 55 from the invariance properties of the Lagrangian density together with the fact that such a Lagrangian depends only on the matter fields and the metric to which they couple.

The previous (standard) formulation is usually called the Jordan frame ${ }^{56}$ Nevertheless, the theory can also be presented differently: in the so-called Einstein frame, one formulates the theory by introducing a transformed metric $\tilde{g}_{a b}$ and deriving the equations from a Lagrangian defined with this metric:

$$
\tilde{L}=\sqrt{-g} \tilde{R}+16 \pi G\left(\tilde{L}_{\Psi}+\tilde{L}_{M}\right)
$$

\footnotetext{
53 For the formulation of the theory see [5]. For further discussion on the interpretation, see 27 and 50 .

54 In the introduction of their paper, Brans and Dicke declare their intention of formulating a theory of gravitation that is more satisfactory form the standpoint of Mach's principle than GR. By this they mean that Mach's idea of the local inertial effects having their origin in the relative accelerated motion of distant masses would be better implemented in their theory.

55 See the previous discussion in 5.2

56 Here I follow the presentation of the theory given in [50].
} 
Where $\tilde{L}_{\Psi}$ is the Lagrangian for a massless scalar field $\Psi=f(\phi)$ and $\tilde{L}_{M}$ is the Lagrangian that depends on the matter fields, $\tilde{g}$ and on $\Psi=f(\phi)$. From this Lagrangian, one can derive the following field equation for $\tilde{g}$.

$$
\tilde{R}_{a b}-\frac{1}{2} \tilde{g}_{a b} \tilde{R}=8 \pi G \tilde{\theta}_{a b}
$$

Being $\tilde{\theta}_{a b}=\tilde{T}_{a b}+\tilde{\Lambda}_{a b}$ an energy-momentum tensor defined from the ordinary tensor for matter $\left(\tilde{T}_{a b}\right)$ and a tensor $\tilde{\Lambda}_{a b}$ associated with $\Psi$. It follows from the equations that $\tilde{\theta}_{a b}$ is automatically conserved with respect to the connection compatible with $\tilde{g}_{a b}$.

In this formulation, then, the gravitational field equations are formally identical to Einstein field equation. It would seem that the conservation condition and, from it, the geodesic motion of free bodies should be derivable with respect to the $\tilde{g}$. But this is not the case: even if the conservation of energymomentum tensor is derivable with respect to the $\widetilde{\nabla}$, from this one cannot infer the geodesic motion of matter. The reason is that matter fields cannot be isolated from coupling to the scalar field, which in the Einstein frame, is treated as a putative matter field. According to the present perspective, this is an illuminating result: it means that it is less natural to treat the scalar field in the Brans-Dicke theory as a regular matter field (as it would be the case in the Einstein frame). The Jordan frame, that regards it as an additional gravitational field provides a much more natural interpretation.

To sum up. In the Jordan frame, matter is coupled to the metric and follows its geodesics, but to derive the conservation condition from the first route one must impose also the satisfaction of the equation for scalar field: so inertial frames would be determined by gravitational plus scalar field (different to GR). In the Einstein frame, putative matter does not follow geodesics of the metric because it cannot be isolated from interaction with the scalar field, indicating that maybe is not appropriate to take such field as simply another matter field. The two situations are compatible with the proposed interpretation of the equivalence principle.

\section{Conclusion}

In GR, locally (in neighbourhoods where curvature terms can be ignored), the laws for all the non-gravitational fields are Poincaré invariant and this invariance coincides with the symmetries of the metric. In the preceding discussion, I have challenged the position that claims that these facts must be considered as brute facts, unexplainable or miraculous in GR. The original claim is motivated by noting that, on the one hand, the geometrical approach is insufficient in providing an explanation of these features of non-gravitational laws (neither does it provide a credible story nor it is able to account for the contingency of the feature) and, on the other, that for the dynamical approach it is a natural extension of its defining motto of regarding the spatiotemporal character of the metric in SR as a consequence of the fact that matter laws are 
Poincaré invariant. Under the perspective of the DA, the chronogeometricity of the metric in GR is a consequence of the coincidental fact of local symmetries of non-gravitational laws being the same as the symmetries of the metric that is governed by Einstein's field equation. This so-called second miracle is also the content of what has been called the strong equivalence principle. So, as a consequence of having a dynamical metric in GR that cannot be conceived as a codification of some features of matter fields, to the miracle of SR - the fact that all matter laws are Poincaré invariant - we must add this second one. If it might already seem too much of a coincidence that all matter laws have the same symmetries in SR, to claim that their coincidence with the symmetries of the dynamical metric finds no explanation in GR might appear as excessive.

My contention is that neither the Poincaré invariance of matter laws in SR nor the coincidence, locally, of this invariance with the symmetries of the metric field in GR must be taken as miraculous; or at least not as much as it has been claimed by defenders of the dynamical perspective. Relativity theory has resources for providing an account of the miracles. The tools for explaining, dissolving or accounting for the miracles can be found in the theory when interpreted in a particular way. And this type of explanation not only works as much in SR as in GR but also meets the perhaps intuitive desideratum of being more complete in GR 57

The central idea of my proposed explanation consists in regarding the facts expressed in the miracles as a consequence of some principles, properly understood, that operate in relativity theory. In particular, in the present paper my focus has been on showing how a certain way of interpreting the equivalence principle does the job of accounting for the miracles. This is performed in three steps. First, I noted that the usual formulations of the equivalence principle are partial in the following sense: they identify it as a requirement on the local behaviour of matter - formulated in different ways - but tend to leave out any reference to gravity. I argue that it is precisely such reference - that by itself can be seen as ambiguous - which gives the physical content to the principle that is essential to explain the miracles. While the usual formulations of the principle are equivalent to the claim of approximate minimal coupling and, therefore, a reformulation of the miracles, the physical version that I endorse states that it is the gravitational interaction which determines locally inertial frames. Second, I showed in what sense the Einstein field equation constrains the coupling of matter to metric and how this can be used to provide specific content to the idea, expressed in the previous step, that gravitational interaction determines inertial frames. Third, I made explicit how having these constraints gives content to the strong equivalence principle in GR (and other theories) and how this accounts for the miracles.

In sum, the facts that are presented as miracles of relativity by [40] are not so if one notices that they can be derived from a version of the equivalence

\footnotetext{
57 By this I mean not the specific explanation of the miracles through the constraints imposed by the Einstein field equation, only available in GR, but the general idea of explaining the symmetries of laws by re-interpreting some of the principles that define the theory. This is the strategy alluded to at the end of the paper.
} 
principle with a particular physical content. The two main posits of such an account for the miracles involve a reinterpretation of the equivalence principle together with the realisation that in GR, and other relativistic theories, the gravitational field equations impose constraints on the behaviour of nongravitational fields. The equivalence principle then, understood as the claim that gravitational interaction determines inertial frames, is satisfied in GR by virtue of the Einstein field equation imposing the constraints that it does impose on the couplings between metric and non-gravitational fields. This is the contingent factual element in the explanation of the miracles: there might not be an interaction that can be employed in the definition of the inertial frames but, given that there is one, this explains that, in a certain regime, non-gravitational laws are locally Poincaré invariant. This is factual, but it is less so than side-stepping this fact and seeing the coincidence of all the symmetries of laws as a mere coincidence. The last option, nevertheless, must be taken when there is no dynamically determined interaction that can be used to derive the constraints, as it is the case in theories with fixed spacetime structures. This difference is crucial to how to understand the relation between GR and SR, on the one hand, and between GR and other theories of gravity, on the other.

The present interpretation can be objected to in the following way 58 the constraints that the Einstein field equation imposes on the non-gravitational laws are non-explanatory or superfluous for accounting for the miracles. The base for such criticism is double: the derivation from the Einstein field equation of something like an operational determination of the inertial frames is contrived and involves hidden assumptions; and the same result that can be derived in GR from the Einstein field equation, can be obtained in GR and other theories without assuming that the metric satisfies any dynamical equations whatsoever, showing that the participation of the gravitational equations is unexplanatory and that the version of the equivalence principle that mentions them is insufficient to account for the miracles. I will respond to these potential objections below.

First, the existence of other assumptions, like energy conditions, for the derivation of the miracles has been proved and is uncontroversial ${ }^{59}$ My claim is that this is not a problem for regarding Einstein's field equation as imposing certain constraints on the behaviour of non-gravitational fields that are valid in a certain regime and under certain approximation. This is in tune with regarding spacetime structure as operationally emerging at certain regime.

Read argues against this kind of explanation by noting, quoting Weatherall's discussion, that Einstein's field equation is neither necessary nor sufficient to derive geodesic motion. As I will expand below, that it is not necessary does not mean that its possibility is not explanatory when it happens. But let us

\footnotetext{
58 [39] considers the possibility of taking into account the constraints imposed by the Einstein field equation, which is part of my proposal, and, as I discuss in the text, dismisses it too quickly. The dismissal is based on Weatherall's discussion of the geodesic theorem in 48, 49.
}

59 See [29], 48], 49. 
look at the question of how the equations not being sufficient affects the project of accounting for the miracles. The grounds for claiming that Einstein's field equation is not sufficient, as Weatherall notes, are that the method to derive geodesic motion "does not establish the energy condition, does not clearly bear on test matter, and cannot establish the relationship between conservation and non-interaction." 60 Taking this for granted, what the miracles claim is that all the non-gravitational laws are locally Poincaré invariant, in neighbourhoods around any point in a regime in which curvature terms can be ignored. So what an explanation of the miracles must assume is that certain conditions obtain: those that would affect certain kind of non-gravitational fields only, or matter considered under some particular conditions. Arguably, this includes non-interacting non-gravitational fields or what we could think of as the default behaviour of matter.

So the relevant question here is not whether a given energy condition is necessary to derive inertial motion from Einstein's field equation, as the point is not whether we have a full explanation of inertial motion by the Einstein field equation only, but whether in GR there is an explanation of certain features of non-gravitational laws (the ones claimed by the miracles). And this can be derived even without assuming that geodesic motion is derivable from the conservation of the energy-momentum tensor, given that certain conditions obtain. So, the key for this type of explanation is that in GR, for a regime in which non-gravitational interactions can be ignored, one can derive from Einstein's field equation the local Poincaré invariance of laws for all individual fields whose energy momentum tensor can be introduced in the r.h.s of the equations. And this can be done. That we should restrict attention to situations in which the conditions obtain is cared for by our formulation of the equivalence principle, in which it is the gravitational interaction which determines this effective spacetime structure. The restriction is correlated to the accuracy of the description of the interaction provided by the theory (GR in this case). If there is a non-gravitational field that affects gravity (interacts gravitationally) or affects the behaviour of any other material field in the way that Einstein's field equation dictates, then it is such that $\nabla_{b} T^{a b}=0$ and this forces that the laws describing its dynamics be locally Poincaré invariant, their invariances coincide with the symmetries of the metric and, given the SDEC, in the smallbody approximation, bodies made of such fields will move on geodesics of that metric. This is basically what the miracles claim, and it can be regarded as a consequence of the Einstein field equation. Should we assume that all matter fields will affect gravity in this way, that they should be taken as potential sources of Einstein's field equation? Well, this is GR's bet on reality and it is the content of the equivalence principle as presented here. It might not be so and this would undermine the adequacy of GR insofar as it is intended that its equations describe a universal interaction. But no one would want to claim

60 49, pp. 12-13 
that this should be seen as a miracle or that there is something conspiratorial in it 61

Second, the possibility of deriving the miracles following a route that does not presuppose the existence of any gravitational field equations, therefore making them non-necessary, is remarkable and essential for the validity of the so-called strong equivalence principle in a wide range of theories. Even more, this is what gives the equivalence principle its general dimension and, let us say, its transcendental character but, at the same time, it is also what makes the principle as non-explanatory, or even empty, insofar as it does not embody a physical interaction through which it acquires specific content ${ }^{62}$ This is, by the way, why if one assumes this partial principle and is not willing to give to it any further justification, the principle can only be understood as the expression of a matter of fact. My claim here is that the existence of the possibility of deriving the miracles without assuming the gravitational equations or, equivalently, the possibility of giving this truncated 63 version of the principle, does not invalidate the explanatory power of having also the other route available. Saying that the miracles are also derivable from other theories with minimal coupling or with a certain matter Lagrangian for which there are no gravitational field equations - implying then that the instances from which they are derivable are nothing else than a re-expression of the mater of fact expressed in the miracles - seems to miss the point of what makes the situation different in GR (and other theories): that it is also derivable from gravitational field equations. This is precisely what gives to it the extra explanatory push.

\footnotetext{
61 One might challenge the claim that this provides any progress with respect to the statement of the miracles. Is it not an assumption that matter enters the Einstein field equation as source in the form of $T^{a b}$ ? Is not this another way of stating the miracles? It is true that the miracles are contained in this interpretation of the equivalence principle and that it can be seen as a mere restatement of the miracles, but my claim is that the derivation from the equivalence principle allows a richer perspective. It is not a question of reducing the number of assumptions, but rather of placing them in a different way. The miracles claim that the coincidence of symmetries of non-gravitational fields is unexplainable. The perspective defended in this paper claims that this can be derived, in GR (although not in other theories) from the equivalence principle properly interpreted. From this point of view, the claim will only be valid for certain kinds of non-gravitational fields: those that can be described as non-interacting and acting as sources of the gravitational field (this, by the way, can be seen as giving content to a certain notion of matter). The hidden assumption in this perspective would then be, if one considers that GR is correct, that all matter meets such conditions. Nonetheless, one can say that this is all the matter that the gravitational interaction, as described by GR, sees. My claim is that this provides a richer look than merely claiming the miracles. Related to this is the fact that principles, beyond stating facts, can be generalised and used as templates for different theories.

62 There is a long tradition, starting with Kant, of understanding some physical principles as neither analytical nor empirical and, in this sense, as constitutive or transcendental. Although related to my proposal, this view on principles is not essential for the core discussion in this paper. [18, [10] are standard references for this view in the context of relativity theory. See [43, 44] for a more recent discussion.

63 I borrow this term from 7 to refer to any version of the principle that imposes certain features to the non-gravitational field equations without stating that this can be derived from the gravitational field equations.
} 
This last point is very relevant for the question about the peculiarities of GR with respect to other theories. If one takes the equivalence principle as a general statement about the couplings of non-gravitational fields to a given metric, then the statement of the miracles seems unavoidable, especially after the realisation of the possibility of theories for which the claim of the local coincidence of the symmetries of the metric and the symmetries of the nongravitational laws is either incorrect or ambiguous. What does this possibility mean from the perspective of the equivalence principle? It means that the claim about the local symmetries of non-gravitational laws is independent from the question about the symmetries of the metric(s) field(s), so the correctness of the first cannot depend on the second (blocking then the explanation of the geometrical perspective). But in some theories like GR or Bekenstein's theory these facts find a deeper explanation. From the perspective gained from GR then we can understand why in the regime in which the Einstein field equation provides a good description of gravity, the truncated version of the equivalence principle holds, thus providing an explanation for it. Our interest in theories that share the same local behaviour of matter can also be seen as a consequence of this. Furthermore, this partially explains the special relativistic character of such laws; partially, because it assumes the signature of the metric that defines the space of kinematically possible models in GR (i.e., that the metric is Lorentzian).

Why should this last feature be assumed? For the DA, this is basically a consequence of non-gravitational laws being Poincaré invariant, which constitutes the first miracle of relativity. Under the perspective endorsed in this paper, this is seen as a consequence of the light principle which, using a similar strategy as the one used for the equivalence principle, can be understood as providing the determination - given the rightness of its implicit empirical claim - of the local causal structure of spacetime ${ }^{64}$ SR then is conceived as a theory that describes the relation between physical events in a regime in which the light principle is valid and that assumes that this posit can be globally extrapolated. GR localises the validity of the light principle by means of the gravitational interaction described by Einstein's field equation, which is encoded in the physical formulation of the equivalence principle that establishes the determination of inertial frames through the gravitational interaction.

\section{Acknowledgements}

I am grateful to two anonymous referees for very helpful comments and suggestions. Research for this article has been supported by the following projects: "Laws, explanation and realism in physical and biomedical sciences" (FFI201676799-P) and "Limits of quantum physics - formalism, interpretation, visualization and aesthetics "(FFI2016-77266-P), Ministerio de Economía y Competitividad (Spain).

\footnotetext{
64 This is developed in more detail in 44, 45. In fact, this is part of a general project for understanding the status of some principles in physical theories.
} 


\section{References}

1. Acuña, P. (2016). Minkowski spacetime and Lorentz invariance: The cart and the horse or two sides of a single coin? Studies in History and Philosophy of Modern Physics, 55, $1-12$.

2. Balashov, I., Janssen, M. (2003). Presentism and relativity. British Journal for the Philosophy of Science, 54, 327-346.

3. Bekenstein, J. D. (2010). Modified gravity as an alternative to dark matter. ArXiv: 1001.3876 [astro-ph.CO]

4. Brading, K. (2001). Symmetries, Conservation Laws, and Noether's Variational Problem. D.Phil. Thesis. University of Oxford.

5. Brans, C. and Dicke, R. H. (1961). Mach's Principle and a Relativistic Theory of Gravitation. Physical Review 124, 3, 925.

6. Brown, H.R. (2005). Physical Relativity: space-time structure from a dynamical perspective. Oxford University Press, Oxford.

7. Brown, H. R. and Pooley, O. The Origin of the Spacetime Metric: Bell's 'Lorentzian Pedagogy', and its Significance in General Relativity, in C. Callender and N. Huggett (eds.), Physics Meets Philosophy at the Planck Scale (pp. 256-276). Cambridge: Cambridge University Press, 2001.

8. Brown, H.R., Pooley, O. (2006). Minkowski space-time: A glorious non-entity. In: D. Dieks (Ed.) The ontology of spacetime (pp. 67-89). Elsevier, Amsterdam.

9. Brown, H., Read, J. (Forthcoming). The dynamical approach to spacetime theories. In E. Knox and A. Wilson (eds.), "The Routledge Companion to Philosophy of Physics" London: Routledge.

10. Disalle, R. (2006). Understanding spacetime: The philosophical development of physics from Newton to Einstein. Cambridge: Cambridge University Press.

11. Doughty, N. (1990). Lagrangian Interaction: An Introduction to Relativistic Symmetry in Electrodynamics and Gravitation. Westview Press.

12. Ehlers, J., Geroch, R. (2004). Equation of motion of small bodies in relativity. Annals of Physics, 309, 232-236.

13. Einstein, A. (1905). Zur elektrodynamik bewegter Ko?rper. Annalen der Physik, 17, 891-921.

14. Einstein, A. (1918). Prinzipielles zur allgemeinen Relativitätstheorie. Annalen der Physik 55: 241-244. In M. Janssen et al. (Eds.) of The collected papers of Albert Einstein (Vol. 7, pp. 37-44). Princeton: Princeton University Press, 2002.

15. Einstein, A. (1919). What is the theory of relativity? London Times, 28 November, Repr. in CPAE, Vol. 7, Doc. 25.

16. Ehlers, J., Pirani, F.A.E., Schild, A. (1972). Republication of: The geometry of free fall and light propagation. Gen Relativ Gravit 44, 1587-1609 (2012). https://doi.org/10.1007/s10714-012-1353-4

17. Fletcher, S. C. (Forthcoming). "Approximate Local Poincaré Spacetime Symmetry in General Relativity" in C. Beisbart, T. Sauer, and C. Wüthrich (eds.), Thinking About Space and Time: 100 Years of Applying and Interpreting General Relativity, Einstein Studies series, vol. 15, Basel: Birkähuser.

18. Friedman, M. (2001). Dynamics of Reason. Stanford: Csli Publications.

19. Frisch, M. (2011). Principle or constructive relativity. Studies in History and Philosophy of Modern Physics 42, 176-183.

20. Geroch, R., Jang, P. S. (1975). Motion of a body in general relativity. Journal of Mathematical Physics, 16(1), 65.

21. Geroch, R., Weatherall, J. O. (2017). The motion of small bodies in space-time. arXiv:1707.04222 [gr-qc].

22. Giovanelli, M. (2013). Erich Kretschmann as a proto-logical-empiricist: Adventures and misadventures of the point-coincidence argument. Studies in History and Philosophy of Modern Physics 44, 115-134.

23. Jacobson, T., Mattingly, D. (2001). Gravity with a dynamical preferred frame. Physical Review D, 64(024028).

24. Janssen, M. (2002). COI stories: Explanation and evidence in the history of science. Perspectives on Science, 10, 457-522. 
25. Janssen, M. (2009). Drawing the line between kinematics and dynamics in special relativity. Studies in History and Philosophy of Modern Physics, 40, 25-52.

26. Knox, E. (2013). Effective spacetime geometry. Studies in History and Philosophy of Modern Physics. 44, 346-356. http://dx.doi.org/10.1016/j.shpsb.2013.04.002.

27. Lehmkuhl, D. (2008). Is Spacetime a Gravitational Field?, in D. Dieks (ed.), The Ontology of Spacetime II. (pp. 1-12) Amsterdam: Elsevier, pp. 83-110.

28. Lehmkuhl, D. (2017). Introduction. In D. Lehmkuhl, G. Schiemann, \& E. Scholz (Eds.), Towards a theory of spacetime theories. Boston, MA: Birkhassuser.

29. Malament, D. (2012a). A remark about the "geodesic principle" in general relativity. In M. Frappier, D. H. Brown, \& R. DiSalle (Eds.), Analysis and interpretation in the exact Sciences: Essays in honour of William Demopoulos (pp. 245-252). New York: Springer.

30. Malament, D. (2012b). Topics in the foundations of general relativity and newtonian gravitation theory. Chicago: University of Chicago Press.

31. Maudlin, T. (2012). Philosophy of Physics Volume I: Space and Time, Princeton: Princeton University Press.

32. Myrvold, W. C. (2017). How could relativity be anything other than physical?, Studies in History and Philosophy of Modern Physics. http://dx.doi.org/10.1016/j.shpsb.2017.05.007

33. Noether, E. (1918). Invariante Variationsprobleme, Nachrichten von der Königlichen Gesellschaft der Wissenschaften zu Göttingen, Mathematisch-physikalische Klasse, 1918, pp. 235-257. (English translation: Invariant Variational Problems in KosmannSchwarzbach, Y. (2011). The Noether Theorems. Springer.

34. Norton, J. (2008). Why constructive relativity fails. British Journal for the Philosophy of Science 59, 821-834.

35. Pauli, W. (1921). Relativitatstheorie, Encycl. Mathematischen Wistentchaften, vol. 5, part 2 (Translated [1958] as Theory of Relativity).

36. Pitts, B. (2019) Space-time Constructivismvs vs.Modal Provincialism: Or, How Special Relativistic Theories Needn't Show Minkowski Chrono- geometry, Studies in History and Philosophy of Modern Physics, pp. 191-198.

37. Pooley, O. (2017). Background Independence, Diffeomorphism Invariance, and the Meaning of Coordinates. In: D. Lehmkuhl, G. Schiemann, E. Scholz, (Eds.) Towards a Theory of Spacetime Theories, Springer.

38. Read, J. (2019). On miracles and spacetime. Studies in History and Philosophy of Modern Physics 65, 103-111. https://doi.org/10.1016/j.shpsb.2018.10.002

39. Read, J. (Preprint). Explanation, geometry and conspiracy in relativity theory.

40. Read, J., Brown, H. Lehmkuhl, D. (2018). Two miracles of general relativiy. Studies in History and Philosophy of Modern Physics 64, 14-25. https://doi.org/10.1016/j.shpsb.2018.03.001

41. Read, J., Menon, T. (2019). The Limitations of Inertial Frame Spacetime Functionalism, Synthese, https://doi.org/10.1007/s11229-019-02299-2.

42. Sus, A. (2014). On the explanation of inertia. Journal for General Philosophy of Science 45, 293-315.

43. Sus, A. (2016). CategorÌas, intuiciones y espacio-tiempo kantiano. Revista de Humanidades de Valparaİso 8, 223-249.

44. Sus, A. (2019) Explanation, analyticity and constitutive principles in spacetime theories. Studies in History and Philosophy of Modern Physics 65, 15-24. https://doi.org/10.1016/j.shpsb.2018.08.002

45. Sus, A. (2020). How to be a realist about Minkowski spacetime without believing in magical explanations- Theoria. An International Journal for Theory, History and Foundations of Science 35, 2, 175-195. https://doi.org/10.1387/theoria.21065

46. Weatherall, J. O. (2011c). On the status of the geodesic principle in Newtonian and relativistic physics. Studies in the History and Philosophy of Modern Physics, 42(4), 276281.

47. Weatherall, J. O. (2012). A brief remark on energy conditions and the Geroch-Jang theorem. Foundations of Physics, 42(2), 209-214.

48. Weatherall, J. O. (2017). Inertial motion, explanation, and the foundations of classical spacetime theories. In D. Lehmkuhl, G. Schiemann, \& E. Scholz (Eds.), Towards a theory of spacetime theories (pp. 13-42). Boston, MA: Birkhassuser. 
49. Weatherall, J. O. (2019). Conservation, inertia, and spacetime geometry. Studies in History and Philosophy of Science Part B: Studies in History and Philosophy of Modern Physics, pp. 144-159.

50. Weinstein, S. (1996). Strange Couplings and Space-Time Structure, Philosophy of Science 63 (Proceedings), pp. S63-S70. 